\title{
A NEW ORDERED COMPACTIFICATION
}

\author{
D.C. KENT \\ Department of Pure and Applied Mathematics \\ Washington State University \\ Pullman, WA 99164 \\ and
}

T.A. RICHMOND

Department of Mathematics

Western Kentucky University

Bowling Green, KY 42101

(Received September 10, 1991 and in revised form Apri1 11, 1992)

\begin{abstract}
A new Wallman-type ordered compactification $\gamma_{0} X$ is constructed using maximal $C Z$ filters (which have filter bases obtained from increasing and decreasing zero sets) as the underlying set. A necessary and sufficient condition is given for $\gamma_{0} X$ to coincide with the Nachbin compactification $\beta_{0} X$; in particular $\gamma_{0} X=\beta_{0} X$ whenever $X$ has the discrete order. The Wallman ordered compactification $\omega_{0} X$ equals $\gamma_{0} X$ whenever $X$ is a subspace of $R^{n}$. It is shown that $\gamma_{0} X$ is always $T_{1}$, but can fail to be $T_{1}$-ordered or $T_{2}$.
\end{abstract}

KEY WORDS AND PHRASES. $C Z$-set, maximal $C Z$-filter, $T_{1}$-ordered space, $T_{2}$-ordered space, Nachbin compactification, Wallman ordered compactification.

1991 AMS SUBJECT CLASSIFICATION CODES. 54F05, 54D35, 54D10

\section{INTRODUCTION.}

L. Nachbin [10] initiated the study of ordered compactifications when he characterized the topological ordered spaces that allow $T_{2}$-ordered compactifications (we call these $T_{3.5}$-ordered spaces), and constructed the largest such $T_{2}$-ordered compactification $\beta_{0} X$ by embedding $X$ in an ordered cube. The Nachbin (or Stone-Cech ordered) compactification $\beta_{o} X$ has been studied and applied by various authors (see, for instance, our odd numbered references). A second ordered (but not necessarily $T_{2}$-ordered) compactification $\omega_{0} X$, called the Wallman ordered compactification, was introduced by Choe and Park [2]. A necessary and sufficient condition for $\omega_{0} X=\beta_{0} X$ was given in [6], and in [8] the separation properties of $\omega_{0} X$ were investigated.

It is well known (e.g., see [4]) that the Stone-Cech compactification $\beta X$ of a $T_{3.5}$ topological space can be described as a Wallman-type compactification using maximal filters of zero sets as the underlying set for the compactification. We have extended this construction to $T_{3.5}$-ordered spaces, and the result is a new ordered compactification which we call $\gamma_{0} X$. This new compactification, like $\beta_{0} X$ and $\omega_{0} X$, has the universal extension property for increasing, continuous maps into compact, $T_{2}$-ordered spaces. With the help of this universal property, we obtain necessary and sufficient conditions for $\gamma_{o} X=\beta_{o} X$; in particular this equality holds when the order of $X$ is discrete. As an alternative approach to constructing $\beta_{0} X, \gamma_{0} X$ is more satisfactory than $\omega_{0} X$, in the sense that $\gamma_{0} X$ and $\beta_{0} X$ coincide on a larger class of spaces than do $\omega_{0} X$ and $\beta_{0} X$. Although we have not yet characterized the class of spaces for which $\gamma_{0} X=\omega_{0} X$, we have shown that this class includes all subspaces of $R^{n}$. This result enables us to show that $\gamma_{0} X$ can exhibit the same "pathological" behavior relative to separation properties that was demonstrated for $\omega_{0} X$ in [8]. 
For example, $\gamma_{0} X$ fails to be $T_{1}$-ordered if $X=R^{n}$ for $n \geq 3$.

It remains an open question whether $\beta_{0} X$ can be described via a Wallman-type ordered compactification for all $T_{3.5}$-ordered spaces $\dot{X}$.

\section{PRELIMINARIES.}

Let $(X, \leq)$ be a poset and let $A$ be a non-empty subset of $X$. Let $d(A)=\{x \in X: x \leq a$ for some $a \in A\}$ and $i(A)=\{x \in X: a \leq x$ for some $a \in A\}$, in case $A=\{x\}$, we write $d(x)$ and $i(x)$ rather than $d(\{x\})$ and $i(\{x\})$. The set $A$ is said to be decreasing (respectively, increasing) if $A=d(A)$ (respectively, $A=i(A)$ ). A set which is either increasing or decreasing is said to be monotone; if $A=d(A) \cap i(A)$, then $A$ is convex. If $f:(X, \leq) \rightarrow(Y, \leq)$ is a function between two posets, then $f$ is increasing (respectively, decreasing) if $x \leq y$ in $X$ implies $f(x) \leq f(y)$ (respectively, $f(y) \leq f(x)$ ) in $Y$.

A topological ordered space $(X, \leq, r)$ is a triple consisting of a poset $(X, \leq)$ and a convex topology $\tau$ on $X ; r$ is convex if the open monotone sets form an open subbase. The term space will always mean topological ordered space, and $(X, \leq, \tau)$ will be shortened to $X$ when there is no ambiguity. Note that every topological space can be regarded as a topological ordered space relative to the discrete order (equality).

Let $E$ be the space $[0,1]$ with its usual order and topology. For an arbitrary space $X$ we denote by $C I^{*}(X)$ (respectively, $C D^{*}(X)$ ) the set of all increasing (respectively, decreasing), continuous maps from $X$ into $E$. An increasing zero set (respectively, decreasing zero set) is a set of the form $f^{-1}(0)$ where $f \in C D^{*}(X)$ (respectively, $f \in C I^{*}(X)$ ). The set of all increasing zero sets (respectively, decreasing zero sets) on $X$ will be designated by $I Z(X)$ (respectively, $D Z(X)$ ). Using standard procedures described in [4], one easily proves the next two propositions.

PROPOSITION 1.1 If $X$ is a space, $f \in C I^{*}(X), g \in C D^{*}(X)$, and $a \in E$, then: $\left(\right.$ a $f^{-1}([0, a]) \in$ $D Z(X) ;\left(\right.$ b) $f^{-1}([a, 1]) \in I Z(X) ;\left(\right.$ c) $g^{-1}([0, a]) \in I Z(X)$, and (d) $g^{-1}([a, 1]) \in D Z(X)$.

PROPOSITION 1.2 For any space $X, I Z(X)$ and $D Z(X)$ are closed under countable intersections and finite unions.

A subset $A$ of a space $X$ is called a $C$-zero set (or $C Z$-set) if there is $B \in I Z(X)$ and $C \in D Z(X)$ such that $A=B \cap C$. Let $C Z(X)$ be the set of all $C Z$-sets on $X$. One easily verifies the following.

PROPOSITION 1.3 (a) $A \in C Z(X)$ iff there is $g \in C I^{*}(X)$ and $h \in C D^{*}(X)$ such that $A=f^{-1}(0)$, where $f=\frac{1}{2}(g+h)$. (b) The set $C Z(X)$ is closed under countable intersections.

It is generally not true that $C Z(X)$ is closed under finite unions; for instance, $[0,1]$ and $[2,3]$ are $C Z$-sets in $R$ whose union is not a $C Z$-set.

By a filter $\mathcal{F}$ on $X$, we always mean a proper set filter (one that does not contain $\theta$ ). The filter on $X$ generated by $\{x\}$, for $x \in X$, will be denoted by $\dot{x}$. If a filter $\mathcal{F}$ has a filter base of increasing zero sets, then $\mathcal{F}$ is called an IZ-filter; $D Z$-filter and $C Z$-filter are defined similarly. For an arbitrary filter $\mathcal{F}$ on $X$, let $I Z(\mathcal{F})$ (respectively, $D Z(\mathcal{F}), C Z(\mathcal{F})$ ) be the filter on $X$ generated by $\mathcal{F} \cap I Z(X)$

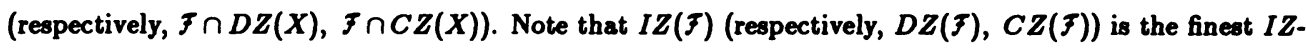
filter (respectively, $D Z$-filter, $C Z$-filter) coarser than $\mathcal{F}$. The next proposition follows from Zorn's Lemma.

PROPOSITION 1.4 If $\mathcal{F}$ is a $C Z$-filter (respectively, $I Z$-filte: $Z Z$-filter), there is a maximal $C Z$-filter (respectively, $I Z$-filter, $D Z$-filter) finer than $\mathcal{F}$.

PROPOSITION 1.5 Let $X, Y$ be spaces and $f: X \rightarrow Y$ an increasing, continuous map.

(a) If $A \in I Z(Y)$ (respectively, $A \in D Z(Y), A \in C Z(Y)$ ), then $f^{-1}(A) \in I Z(X)$ (respectively, $f^{-1}(A) \in$ $\left.D Z(X), f^{-1}(A) \in C Z(X)\right)$.

(b) If $\mathcal{F}$ is a filter on $X$, then $I Z(f(\mathcal{F})) \leq f(I Z(\mathcal{F})), D Z(f(\mathcal{F})) \leq f(D Z(\mathcal{F}))$, and $C Z(f(\mathcal{F})) \leq f(C Z(\mathcal{F}))$. 
PROOF. (a) If $A \in I Z(Y)$, then $A=g^{-1}(0)$, for $g \in C D^{*}(Y)$. Then $f^{-1}(A)=(g \circ f)^{-1}(0)$, where $g \circ f \in C D^{*}(X)$, and so $f^{-1}(A) \in I Z(X)$. The other cases are similar. (b) follows easily from (a).

A space $X$ is defined to be $T_{1}$-ordered if, for each $x \in X, i(x)$ and $d(x)$ are closed sets. A space $X$ is $T_{2}$-ordered if, whenever $x \geq y$ in $X$, there is an increasing neighborhood $U$ of $x$ and a decreasing neighborhood $V$ of $y$ such that $U \cap V=\emptyset$; equivalently, $(X, \leq, \tau)$ is $T_{2}$-ordered if the order $\leq$ is a closed subset of $X \times X$. A space $X$ is $T_{3.5}$-ordered if it satisfies the following conditions: (1) If $x \in X, A$ is a closed subset of $X$, and $x \notin A$, then there is $f \in C I^{*}(X)$ and $g \in C D^{*}(X)$ such that $f(x)=g(x)=0$ and $f(y) \vee g(y)=1$ for $y \in A$; (2) If $x \notin y$ in $X$, there is an $f \in C I^{*}(X)$ such that $f(y)=0$ and $f(x)=1$. The $T_{3.5}$-ordered spaces are precisely the subspaces of compact, $T_{2}$-ordered spaces (see [10]). A space $X$ is defined to be $T_{4}$-ordered if it is $T_{1}$-ordered and, whenever $A$ and $B$ are disjoint closed subsets with $A$ decreasing and $B$ increasing, there are disjoint open sets $U$ and $V$, the former decreasing, the latter increasing, such that $A \subseteq U$ and $B \subseteq V$. Note that: compact and $T_{2}$-ordered $\Rightarrow T_{4}$-ordered $\Rightarrow T_{3.5}$-ordered $\Rightarrow T_{2}$-ordered $\Rightarrow T_{1}$-ordered. Also observe that $T_{1}$-ordered $\Rightarrow T_{1}, T_{2}$-ordered $\Rightarrow T_{2}$, and $T_{3.5}$-ordered $\Rightarrow T_{3.5}$ (i.e., completely regular and $T_{1}$ ); it is not true, however, that $T_{4}$-ordered $\Rightarrow T_{4}$.

In the remainder of this section we examine some properties of $T_{3.5}$-ordered spaces, with special emphasis on the role played by $C Z$-sets.

PROPOSITION 1.6 Let $X$ be a $T_{3.5}$-ordered space. Let $x \in X$, and let $\mathcal{V}(x)$ be the filter of néighborhoods of $x$.

(a) $\nu(x)=C Z(\nu(x))$

(b) $\mathcal{V}(x)$ has an open base of sets of the form $(X-A) \cap(X-B)$, where $A \in C Z(X)$ and $B \in D Z(X)$.

(c) $C Z(X)$ is a closed subbase for $X$.

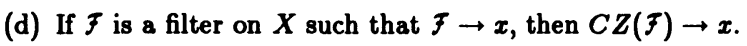

PROOF. (a) Let $V$ be an open neighborhood of $x$. Then there are $f \in C I^{*}(X)$ and $g \in C D^{*}(X)$ such that $f(x)=g(x)=0$ and $f(y) \vee g(y)=1$ if $y \in X-V$. Then $f^{-1}\left(\left[0, \frac{1}{2}\right]\right) \cap g^{-1}\left(\left[0, \frac{1}{2}\right]\right)$ is a $C Z$-set neighborhood of $x$ which is a subset of $V$.

(b) Let $f, g$, and $V$ be as in the proof of (a). If $B=f^{-1}(1)$ and $A=g^{-1}(1)$, then $A \in D Z(X)$, $B \in I Z(X)$, and $x \in(X-A) \cap(X-B) \subseteq V$.

(c) and (d) follow immediately from (b) and (a), respectively.

PROPOSITION 1.7 In a $T_{3.5}$-ordered space $X$, the following statements are equivalent: (a) $x \leq y$; (b) $I Z(\dot{x}) \leq \dot{y}$; (c) $D Z(\dot{y}) \leq \dot{x}$.

PROOF. It is obvious that $(a) \Rightarrow(b)$. To show $(b) \Rightarrow(a)$, suppose $y$ is in each member of $I Z(X)$ containing $x$, but $x \notin y$. Then there is $f \in C I^{*}(X)$ such that $f(y)=0$ and $f(x)=1$. Thus $y \notin f^{-1}(1)$, but $f^{-1}(1)$ is a member of $I Z(X)$ containing $x$. This establishes that $(a) \Leftrightarrow(b)$, and $(c) \Leftrightarrow(a)$ follows by a dual argument.

In the next section we shall construct a compactification based on maximal $C Z$-filters. The next two propositions will be useful in this endeavor.

PROPOSITION 1.8 If $X$ is a $T_{3.5}$-ordered space and $x \in X$, the $C Z(\dot{x})$ is the unique maximal $C Z$ filter on $X$ coarser than $\dot{x}$.

PROOF. We already know that $C Z(\dot{x})$ is the finest $C Z$-filter coarser than $\dot{x}$. Suppose $\mathcal{G}$ is a $C Z$-filter and $C Z(\dot{x})<\mathcal{G}$. Then there is a $C Z$-set $G \in \mathcal{G}$ such that $x \in X-G$. By Proposition 1.6(a), there is a $C Z$-neighborhood $H$ of $x$ such that $H \subseteq X-G$. Since $H \in C Z(\dot{x})$, the assumption that $C Z(\dot{x})<\mathcal{G}$ is 
contradicted, and it follows that $C Z(\dot{x})$ is a maximal $C Z$-filter. It is obviously the only maximal $C Z$-filter coarser than $\dot{x}$.

PROPOSITION 1.9 Let $f: X \rightarrow Y$ be a continuous, increasing map, where $X$ is $T_{3.5}$-ordered and $Y$ is compact and $T_{2}$-ordered. If $M$ is a maximal $C Z$-filter on $X$, there is a unique point $y_{M} \in Y$ such that $f(\mathcal{M}) \rightarrow y_{\mathcal{M}}$ in $Y$.

PROOF. Let $\mathcal{F}$ be an ultrafilter on $X$ such that $M \leq \mathcal{F}$. Since $Y$ is compact and $T_{2}$, there is a unique point $y_{M}$ in $Y$ such that $f(f) \rightarrow y_{M}$. Because $M$ is a maximal $C Z$-filter, $C Z(f) \leq M$, and $f(\mathcal{M}) \geq f(C Z(\mathcal{F})) \geq C Z(f(\mathcal{F}))$ follows by Proposition 1.5. But $f(\xi) \rightarrow y_{M}$ implies $C Z(f(\mathcal{F})) \rightarrow y_{M}$ by Proposition 1.6(d), and therefore $f(M) \rightarrow y_{M}$.

\section{THE COMPACTIFICATION $\gamma_{0} X$}

Throughout this section, we assume that $X$ is a $T_{3.5}$-ordered space. An ordered compactification $(Y, \sigma)$ of $X$ is a pair consisting of a compact space $Y$ and a map $\sigma: X \rightarrow Y$ such that $\sigma$ is both a topological and an order embedding of $X$ into $Y$ such that $\sigma(X)$ is dense in $Y$. In this section, we shall construct an ordered compactification $\left(\gamma_{0} X, \psi\right)$ of $X$ and establish some of its basic properties.

Let $\tilde{X}$ be the set of all maximal $C Z$-filters on $X$. By Proposition 1.8, these include all filters of the form $C Z(\dot{x})$, where $x \in X$. A relation $\lesssim$ on $\tilde{X}$ is defined as follows: If $\mathcal{M}, \mathcal{N} \in \tilde{X}$, then $\mathcal{M} \lesssim \mathcal{N}$ iff $I Z(\mathcal{M}) \leq \mathcal{N}$ and $D Z(\mathcal{N}) \leq \mathcal{M}$.

PROPOSITION $2.1 \quad(\tilde{X}, \lesssim)$ is a poset.

PROOF. It is clear that $\lesssim$ is reflexive and transitive. If $M \leqslant N$ and $N \leqslant M$, then $I Z(\mathcal{N}) \leq M$ and $D Z(\mathcal{N}) \leq \mathcal{M}$. Since $\mathcal{N}$ is a $C Z$-filter, $I Z(\mathcal{N}) \vee D Z(N)=\mathcal{N}$, and so $N \leq \mathcal{M}$. It is also true that $I Z(\mathcal{M}) \leq \mathcal{N}$ and $I Z(\mathcal{N}) \leq M$; therefore $\mathcal{M} \leq \mathcal{N}$, and we conclude that $M=\mathcal{N}$.

PROPOSITION $2.2 x \leq y$ in $X$ iff $C Z(\dot{x}) \lesssim C Z(\dot{y})$ in $\tilde{X}$.

PROOF. If $x \leq y$, then by Proposition 1.7, $I Z(\dot{x})=I Z(C Z(\dot{x})) \leq \dot{y}$, which implies $I Z(C Z(\dot{x})) \leq$ $C Z(\dot{y})$. Likewise, $D Z(\dot{y}) \leq \dot{x}$, which implies $D Z(C Z(\dot{y})) \leq C Z(\dot{x})$. Thus $C Z(\dot{x}) \leqq C Z(\dot{y})$. This reasoning is reversible.

For an arbitrary, non-empty subset $A$ of $X$, we define $\tilde{A}=\{\mathcal{M} \in \tilde{X}: A \in \mathcal{M}\}$.

PROPOSITION 2.3 Let $A, B \in C Z(X)$.

(a) $\tilde{A} \cap \tilde{B}=\tilde{A} \widetilde{\cap B}$.

(b) $\tilde{A} \cup \tilde{B}=\tilde{A} \widetilde{\cup}$.

(c) $\tilde{\boldsymbol{X}}-\tilde{\boldsymbol{A}}=\tilde{\boldsymbol{X}}-\boldsymbol{A}$.

(d) If $A \in I Z(X)$, then $\tilde{A}$ is an increasing set in $\tilde{X}$.

(e) If $A \in D Z(X)$, then $\tilde{A}$ is a decreasing set in $\tilde{X}$.

PROOF. All of the ascertions of this proposition are routine, and we shall verify only (d). If $M \in \tilde{A}$ and $\mathcal{M} \lesssim \mathcal{N}$, then $I Z(\mathcal{M}) \leq \mathcal{N}$. Since $A \in \mathcal{M}$ and $A \in I Z(X), A \in I Z(\mathcal{M})$, and so $A \in \mathcal{N}$. Thus $\mathcal{N} \in \tilde{A}$, and $\tilde{A}$ is an increasing set.

We next define $\psi: X \rightarrow \tilde{X}$ by $\psi(x)=C Z(\dot{x})$, for all $x \in X$. By Proposition 2.2, $\psi$ is an order embedding of $X$ in $\tilde{X}$. We omit the routine proof of the next proposition.

PROPOSITION 2.4 (a) For any $A \subseteq X, \psi^{-1}(\tilde{A}) \subseteq A$.

(b) If $A \in C Z(X)$, then $\psi^{-1}(\tilde{A})=A$ and $\psi^{-1}(\widetilde{X-A})=X-A$. 
Let $\tilde{\tau}$ be the topology on $\tilde{X}$ with closed subbase $\{\tilde{A}: A \in C Z(X)\}$. From the two preceding propositions, it follows that $\tilde{\tau}$ has an open subbase of monotone open sets; thus $(\tilde{X}, \lesssim, \tilde{\tau})$ is a topological ordered space. Let $\gamma_{0} X=(\tilde{X}, \lesssim, \tilde{\tau})$.

THEOREM 2.5 For any $T_{3}$-ordered space $X,\left(\gamma_{o} X, \psi\right)$ is an ordered compactification for $X$ whose topology is $T_{1}$.

PROOF. First note that $\psi: X \rightarrow \gamma_{o} X$ is a topological embedding by Propositions 1.6(c) and 2.4(b); $\psi$ is also an order embedding, as we observed previously.

To show that $\gamma_{0} X$ is compact, it is sufficient to show that any collection $C=\left\{\tilde{A}_{i}: A_{i} \in C Z(X), i \in I\right\}$ of subbasic closed sets in $\gamma_{0} X$ with the finite intersection property has a non-empty intersection. If $A=\left\{A_{i}: i \in I\right\}$, then $A$ has the finite intersection property by Proposition 2.3(a). Let $\mathcal{M}$ be any maximal $C Z$-filter containing $A$; then $M \in \cap C$.

To show that $\gamma X_{0}$ is $T_{1}$, let $\mathcal{M}, \mathcal{N}$ be two distinct maximal $C Z$-filters on $X$. Then there are disjoint $C Z$-sets $M \in M$ and $N \in \mathcal{N}$. It follows that $\widetilde{X-N}$ is a neighborhood of $\mathcal{M}$ not containing $\mathcal{N}$, and $X \widetilde{-M}$ is a neighborhood of $\mathcal{N}$ not containing $\mathcal{M}$.

Finally, if $\mathcal{M} \in \tilde{X}$, then $\psi(\mathcal{M})$ converges to $\mathcal{M}$ in $\gamma_{0} X$, and therefore $\psi(X)$ is dense in $\gamma_{0} X$.

The next theorem shows that $\gamma_{0} X$ has the same universal extension property as $\omega_{o} X$ and $\beta_{o} X$.

THEOREM 2.6 Let $X$ be a $T_{3.5}$-ordered space, $Y$ a compact, $T_{2}$-ordered space, and $f: X \rightarrow Y$ be a continuous, increasing map. Then there is a unique continuous, increasing map $\tilde{f}: \gamma_{0} X \rightarrow Y$ such that the diagram below commutes.

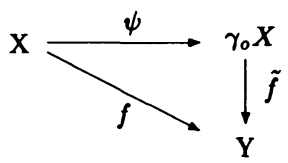

PROOF. Let $\tilde{f}: \gamma_{0} X \rightarrow Y$ be defined by $\tilde{f}(\mathcal{M})=y_{\mathcal{M}}$, where $y_{\mathcal{M}}$ is defined in Proposition 1.9. We first show that $\tilde{f}$ is increasing. Let $\mathcal{M} \lesssim \mathcal{N}$ in $\tilde{X}$; then $D Z(\mathcal{N}) \leq \mathcal{M}$.

Suppose $y_{\mathcal{M}} \mathbb{Z} y_{\mathcal{N}}$ in $Y$. Then there is $g \in C I^{*}(Y)$ such that $g\left(y_{\mathcal{M}}\right)=1$ and $g\left(y_{\mathcal{N}}\right)=0$. Thus $y_{\mathcal{N}} \in g^{-1}\left(\left[0, \frac{1}{3}\right]\right) \in D Z(f(\mathcal{N}))$, since $f(\mathcal{N}) \rightarrow y_{\mathcal{N}}$ in $Y$. But $g^{-1}\left(\left[\frac{2}{3}, 1\right]\right) \in f(\mathcal{M})$, since $f(\mathcal{M}) \rightarrow y_{\mathcal{M}}$, and therefore $f(\mathcal{M}) \succeq D Z(f(\mathcal{N}))$. However, $D Z(\mathcal{N}) \leq \mathcal{M}$ implies $D Z(f(\mathcal{N})) \leq f(D Z(\mathcal{N})) \leq f(\mathcal{M})$ follows by Proposition 1.5. This contradiction establishes that $y_{\mathcal{M}} \leq y_{\mathcal{N}}$, and so $\tilde{f}$ is increasing.

We next show that $\tilde{f}$ is continuous. Let $M \in \gamma_{0} X$ and let $A$ be a $C Z$-neighborhood of $y_{M}$ in $Y$. From the fact that $f(\mathcal{M}) \rightarrow y_{\mathcal{M}}$, we deduce that $\mathcal{M} \in f^{-1}(A)$, and it is easy to see that $\tilde{f}\left(f^{-1}(A)\right) \subseteq A$. It remains to show that $f^{-1}(A)$ is a neighborhood of $\mathcal{M}$ in $\gamma_{0} X$. For this purpose, we employ Proposition 1.6(b) to obtain $C \in D Z(Y)$ and $D \in I Z(Y)$ such that $y_{\mathcal{M}} \in(Y-C) \cap(Y-D) \subseteq A$. Since $(Y-C) \cap(Y-D) \in f(\mathcal{M})$, it follows that $\mathcal{M} \in\left(\tilde{X}-f^{-1}(C)\right) \cap\left(\tilde{X}-f^{-1}(D)\right)$. The latter set is open in $\gamma_{0} X$ and a subset of $f^{-1}(A)$. This establishes that $f^{-1}(A)$ is a neighborhood of $\mathcal{M}$ which maps into $A$, and the proof is complete.

THEOREM 2.7 Let $X$ be $T_{3.5}$-ordered. Then $\gamma_{0} X=\beta_{o} X$ iff the following conditions hold:

(1) If $M \in I Z(X), N \in C Z(X)$, and $M \cap N=\emptyset$, then there is $h \in C I^{*}(X)$ such that $h(N)=0$ and $h(M)=1$.

(2) If $M \in D Z(X), N \in C Z(X)$, and $M \cap N=\emptyset$, then there is $h \in C D^{*}(X)$ such that $h(M)=0$ and $h(N)=1$.

PROOF. Since $\beta_{o} X$ is the largest $T_{2}$-ordered compactification of $X$, Theorem 2.6 implies that $\gamma_{o} X=\beta_{o} X$ iff $\gamma_{o} X$ is $T_{2}$-ordered. Thus the proof will be achieved by showing that the specified conditions are necessary and sufficient in order for $\gamma_{0} X$ to be $T_{2}$-ordered. 
Assume that $\gamma_{0} X$ is $T_{2}$-ordered and let $M$ and $N$ be as indicated in (1). By Proposition 2.3(a), $\tilde{M} \cap \tilde{N}=\emptyset$, and $\tilde{M}$ and $\tilde{N}$ are both closed subsets of $\gamma_{0} X$. Furthermore, $\tilde{M}$ is increasing in $\gamma_{0} X$ by Proposition 2.3(d). Let $d(\tilde{N})$ denote the decreasing hull of $\tilde{N}$ in $\gamma_{0} X$. Then $d(\tilde{N})$ is closed by Proposition 4, page 44, $[10]$, and $d(\tilde{N}) \cap \tilde{M}=0$. By Theorem 1, page 30, [10], there is $g$ in $C I^{*}\left(\gamma_{o} X\right)$ such that $g(\mathcal{M})=0$ if $\mathcal{M} \in d(\tilde{N})$ and $g(\mathcal{M})=1$ if $\mathcal{M} \in \tilde{M}$. Setting $h=g \circ \psi$, we obtain (1). A similar argument establishes (2).

Conversely, assume the two conditions, and let $\mathcal{M}, \mathcal{N}$ be elements of $\gamma_{0} X$ such that $\mathcal{M} \nsubseteq \mathcal{N}$. Then either $I Z(\mathcal{M}) \notin \mathcal{N}$ or $D Z(\mathcal{N}) \& \mathcal{M}$. If $I Z(\mathcal{M}) \& \mathcal{N}$, then (because $\mathcal{N}$ is a maximal $C Z$-filter) there is

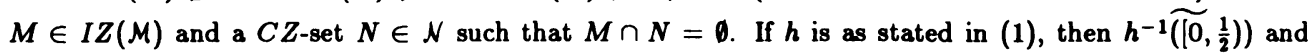
$h^{-1} \widetilde{\left(\left(\frac{1}{2}, 1\right]\right)}$ are disjoint open neighborhoods of $\mathcal{N}$ and $M$ respectively, the former decreasing and the latter increasing. If $D Z(\mathcal{N}) \& \mathcal{M}$, we can apply (2) to achieve the same result.

If $X$ has the discrete order, conditions (1) and (2) of Theorem 2.7 reduce to the statement that disjoint zero sets in $X$ are "completely separated" in the sense of [4]. Since this is true for any $T_{3.5}$ space, we conclude that $\gamma_{0} X=\beta_{0} X=\beta X$ whenever $X$ is a $T_{3.5}$-ordered space with the discrete order.

As we shall see in the next section, there are simple examples of $T_{3.5}$-ordered spaces for which $\gamma_{0} X$ is not $T_{2}$-ordered. In this case, we may be interested to know when $\gamma_{0} X$ satisfies the weaker separation properties " $T_{2}$ " or " $T_{1}$-ordered". This section concludes with two theorems pertaining to this problem. Examples showing that $\gamma_{0} X$ need not satisfy these latter separation axioms are also provided in the next section.

THEOREM 2.8 Let $X$ be a $T_{3.5}$-ordered space. Then $\gamma_{0} X$ is $T_{2}$ iff, for each ultrafilter $\mathcal{F}$ on $X$, there is a unique maximal $C Z$-filter $\mathcal{M}$ on $X$ such that $C Z(\mathcal{F}) \leq \mathcal{M}$.

PROOF. Assume $\gamma_{o} X$ is $T_{2}$ and let $\mathcal{F}$ be an ultrafilter on $X$. Then $\psi(\xi)$ converges to some $M \in \gamma_{o} X$, where $\mathcal{M}$ is a maximal $C Z$-filter on $X$. It must be true that $C Z(\mathcal{F}) \leq \mathcal{M}$; otherwise $\mathcal{M}$ and $C Z(\mathcal{F})$ would contain disjoint $C Z$-sets $M$ and $A$, and $\widetilde{X-A}$ would be a neighborhood of $\mathcal{M}$ in $\gamma_{0} X$ not belonging to $\psi(\mathcal{F})$. If there were another maximal $C Z$-filter $\mathcal{N}$ finer than $C Z(\mathcal{F})$, then $\psi(\mathcal{F})$ would also converge to $\mathcal{N}$ in $\gamma_{0} X$, contradicting the assumption that $\gamma_{0} X$ is $T_{2}$. Thus $M$ is the unique maximal $C Z$-filter such that $C Z(\xi) \leq \mathcal{M}$.

Conversely, assume that $\gamma_{0} X$ is not $T_{2}$; then there is a filter $A$ on $\gamma_{0} X$ converging to distinct elements $\mathcal{M}$ and $\mathcal{N}$ in $\gamma_{0} X$. Let $\mathcal{F}$ be an ultrafilter on $X$ containing the filter base $\{A \subseteq X: \tilde{A} \in A\}$. One easily verifies that $\psi(\xi)$ converges to both $\mathcal{M}$ and $\mathcal{N}$ in $\gamma_{0} X$. This implies, as in the preceding paragraph, that $M$ and $\mathcal{N}$ are both maximal $C Z$-filters finer than $C Z(\mathcal{F})$, which contradicts the uniqueness condition.

THEOREM 2.9 Let $X$ be a $T_{3.5}$-ordered space such that, for each $A \in C Z(X), i(A) \in I Z(X)$ and $d(A) \in D Z(X)$. Then $\gamma_{0} X$ is $T_{1}$-ordered.

PROOF. For $S \subseteq \gamma_{0} X$, let $i_{\gamma}(S)$ denote the increasing hull of $S$ and $c l_{\gamma} S$ the closure of $S$ in $\gamma_{0} X$. We will show that for arbitrary $\mathcal{M} \in \gamma_{0} X$, that $c l_{\gamma}\left(i_{\gamma}(\mathcal{M})\right)=i_{\gamma}(\mathcal{M})$, and hence $i_{\gamma}(\mathcal{M})$ is closed in $\gamma_{0} X$. The dual argument establishes that $d_{\gamma} M$ is also closed.

First, observe that if $\mathcal{N} \in \operatorname{cl}_{\gamma}\left(i_{\gamma}(\mathcal{M})\right)$, then for each $A \in C Z(X)$ such that $\mathcal{N} \in X \widetilde{X}$, there is $\mathcal{X} \in i_{\gamma}(\mathcal{M})$ such that $\mathcal{X} \in \widetilde{X-A}$. In other words, if $\mathcal{N} \in c l_{\gamma}\left(i_{\gamma}(\mathcal{M})\right)$, then for each $A \in C Z(X)$ such that $A \notin \mathcal{N}$, there is $\mathcal{H} \in \gamma_{0} X$ such that $M \lesssim \mathcal{H}$ and $A \notin X$.

Let $\mathcal{N} \in c l_{\gamma}\left(i_{\gamma}(\mathcal{M})\right)$. If $\mathcal{N} \notin i_{\gamma}(\mathcal{M})$, then $\mathcal{M} \mathbb{N}$, and so either $I Z(\mathcal{M}) \not \mathcal{N}$ or $D Z(\mathcal{N}) \nsubseteq \mathcal{M}$. Assume the former; then there is $M \in \mathcal{M} \cap I Z(X)$ such that $M \notin \mathcal{N}$. But $\mathcal{N} \in \operatorname{cl}_{\gamma}\left(i_{\gamma}(\mathcal{M})\right)$ implies there is $\mathcal{K} \in i_{\gamma}(\mathcal{M})$ such that $M \notin \mathcal{K}$. However $\mathcal{M} \lesssim \nVdash$ implies $I Z(\mathcal{M}) \leq K$, a contradiction. On the other hand, suppose $D Z(\mathcal{N}) \notin \mathcal{M}$. Since $\mathcal{M}$ is a maximal $C Z$-filter, there is a $C Z$-set $M \in \mathcal{M}$ and $N \in \mathcal{N} \cap D Z(X)$ such that $M \cap N=\emptyset$, and hence $N \cap i(M)=0$. But by assumption, $i(M) \in I Z(X)$, and so $i(M) \in I Z(M)$. Again, $\mathcal{N} \in c l_{\gamma}\left(i_{\gamma}(\mathcal{M})\right)$ implies there is $\not \gtrsim \mathcal{M}$ such that $i(M) \notin \mathcal{H}$. However $i(M) \in I Z(\mathcal{M}) \leq \not$ is again a contradiction. We therefore conclude that $i_{\gamma}(M)$ is closed in $\gamma_{0} X$. 
3. $\gamma_{o} X$ AND $\omega_{o} X$

The Wallman ordered compactification $\left(\omega_{0} X, \varphi\right)$ of a $T_{1}$-ordered space $X$ was introduced by Choe and Park [2] in 1979. In this section we find conditions under which $\gamma_{0} X=\omega_{0} X$; this leads to examples showing that $\gamma_{0} X$ can fail, in various ways, to preserve the separation properties $T_{2}, T_{2}$-ordered, and $T_{1}$-ordered.

The construction of $\omega_{0} X$ and a discussion of its properties can be found in [8]. Here, we review only a few relevant facts. Although $\gamma_{0} X$ can be defined for any $T_{1}$-ordered space $X$, we shall assume, as in the preceding section, that $X$ is $T_{3.5}$-ordered, since it is only for such spaces that $\gamma_{0} X$ and $\omega_{0} X$ can be compared.

If $A$ is any non-empty subset of $X$, let $I(A)$ denote the smallest closed, increasing set containing $A$ and $D(A)$ the smallest closed, decreasing overset of $A$. $A$ is said to be a c-set if $A=I(A) \cap D(A)$. A space $X$ is called a $c$-space if, for every c-set $A \subseteq X, i(A)=I(A)$ and $d(A)=D(A)$. A filter on $X$ with a base of $c$-sets is called a $c$-filter. The underlying set for $\omega_{0} X$ is the set of all maximal $c$-filters on $X$. Indeed, the constructions of $\omega_{0} X$ and $\gamma_{0} X$ are very similar, with the c-sets playing the same role in the former that the $C Z$-sets play in the latter. In particular, if every $c$-set in $X$ is a $C Z$-set, then $\omega_{0} X=\gamma_{0} X$. Thus the following proposition is obvious.

PROPOSITION 3.1 If every increasing closed set in $X$ is in $I Z(X)$ and every decreasing closed set in $X$ is in $D Z(X)$, then $\omega_{0} X=\gamma_{0} X$.

Another useful fact, proved in [6], is the following.

PROPOSITION 3.2 A space $X$ has the property that $\omega_{0} X=\beta_{0} X$ iff $X$ is a $T_{4}$-ordered $c$-space.

THEOREM 3.3 If $X$ is a $T_{4}$-ordered space such that, for any sets $F, G$ in $C Z(X), I(F) \cap G=\emptyset$ implies $I(F) \cap D(G)=\emptyset$ and dually, then $\gamma_{0} X=\beta_{0} X$.

PROOF. We show that, under the given assumptions, $X$ satisfies conditions (1) and (2) of Theorem 2.7. To verify (1), let $M \in I Z(X)$ and $N \in C Z(X)$ be disjoint. Since $I(M)=M$, it follows by our assumption that $M \cap D(G)=\emptyset$. Thus we can apply Nachbin's generalization of Urysohn's Lemma (see Theorem 1, page 30, [10]) to obtain $f \in C I^{*}(X)$ such that $f(M)=1$ and $f(D(G))=0$. This establishes condition (1); the proof of (2) is similar.

COROLLARY 3.4 If $X$ is a $T_{3.5}$-ordered space such that $\omega_{0} X=\beta_{0} X$, then $\omega_{0} X=\gamma_{0} X$.

PROOF. If $\omega_{0} X=\beta_{0} X$, then, by Proposition 3.2, $X$ is a $T_{4}$-ordered $c$-space. Every such space clearly satisfies the requirements of Theorem 3.3, and so the conclusion follows.

A $T_{2}$-ordered space whose underlying partial order is a total (or linear) order is called a totally ordered space. It is shown in [7] that $\omega_{o} X=\beta_{0} X$ for any totally ordered space $X$.

COROLLARY 3.5 If $X$ is a totally ordered space, then $\omega_{0} X=\gamma_{0} X=\beta_{0} X$.

THEOREM 3.6 Let $X$ be a subspace of $R^{n}$. Then $\gamma_{0} X=\omega_{0} X$.

PROOF. In view of Proposition 3.1, it is sufficient to show that each closed, decreasing subset of $X$ is in $D Z(X)$ and each closed, increasing subset of $X$ is in $I Z(X)$.

We begin by defining (in the terminology of [3]) a quasi-pseudo-metric $\rho$ on $X$ defined as iollows: If $x=\left(x_{1}, \cdots, x_{n}\right), y=\left(y_{1}, \cdots, y_{n}\right)$, then $\rho(x, y)=\left(y_{1}-x_{1}\right) \vee 0+\cdots+\left(y_{n}-x_{n}\right) \vee 0$. If $A$ is a non-empty, closed, decreasing subset of $X$, we define $\rho_{A}: X \rightarrow[0, \infty)$ as follows: $\rho_{A}(x)=\inf \{\rho(y, x): y \in A\}$. Finally, let $h_{A}: X \rightarrow E$ be defined by $h_{A}=\rho_{A} \wedge 1$. It follows that $h_{A} \in C I^{*}(X)$ and $h_{A}^{-1}(0)=A$. Thus $A \in D Z(X)$. The dual argument shows that any closed, increasing subset of $X$ is in $I Z(X)$.

It is shown in Theorem $3 . \overrightarrow{4}$ of $[8]$ that $\omega_{0} R^{n}=\beta_{0} R^{n}$ iff $n \leq 2$; this yields the following consequence of Theorem 3.6. 
COROLLARY $3.7 \gamma_{o} R^{n}=\beta_{o} R^{n}$ iff $n \leq 2$.

We recall two examples from [8] involving subspaces of $R^{2}$ in which $\omega_{0} X$, and hence also $\gamma_{0} X$, fail to exhibit basic separation properties. Let $S=\{(x, y):-1 \leq x \leq 1,-1 \leq y \leq 1\}$ be a subspace of $R^{2}$. In Example 3.6 of $[8]$, the subspace $X_{1}=S-\{(0,0)\}$ of $R^{2}$ has the property that $\gamma_{0} X_{1}$ is neither $T_{1}$-ordered nor $T_{2}$. In Example 3.7 of $[8]$, the subspace $X_{2}=S-\{(0, y):-1 \leq y \leq 1$ and $y \neq 0\}$ has the property that $\gamma_{0} X_{2}$ is $T_{2}$ but not $T_{1}$-ordered. We do not know of a space $X$ for which $\gamma_{0} X$ is $T_{1}$-ordered but not $T_{2}$.

As a final example, recall that if $X$ is a $T_{3.5}$-ordered space with the discrete order, then $\gamma_{0} X=\beta_{0} X$. If, in addition, $X$ is chosen not to be $T_{4}$, then $\omega_{0} X$ (which in this case is the ordinary Wallman compactification) fails to be $T_{2}$, and consequently $\omega_{0} X \neq \gamma_{0} X$.

\section{UNSOLVED PROBLEMS.}

(1) Find necessary and sufficient conditions on a space $X$ for $\gamma_{0} X$ to be $T_{1}$-ordered.

(2) Find conditions on a space $X$ which are necessary and sufficient for $\gamma_{0} X=\omega_{0} X$.

(3) Determine whether $\gamma_{0} R^{3}$ is $T_{2}$.

(4) Find a $T_{3.5}$-ordered space $X$ for which $\omega_{0} X, \beta_{0} X$, and $\gamma_{0} X$ are mutually non-equivalent.

(5) Determine whether $\beta_{o} X$ can be represented as a Wallman-type ordered compactification.

\section{REFERENCES}

[1] J. Blatter, "Order Compactification of Totally Ordered Spaces," J. Approx. Theory 13 (1975) 56-65.

[2] T. H. Choe and S. Park, "Wallman's Type Order Compactification," Pacific J. Math. 82 (1979) 339-347.

[3] P. Fletcher and W. Lindgren, Quasi-Uniform Spaces, Lecture Notes in Pure and Applied Mathematics, Vol. 77, Marcel Dekker, Inc., New York (1982).

[4] L. Gillman and M. Jerison, Rings of Continuous Functions, Van Nostrand, Princeton (1960).

[5] G. Hommel, "Increasing Radon Measures on Locally Compact Ordered Spaces," Rendiconti Mathematica 9 (1976) 85-117.

[6] D. C. Kent, "On the Wallman Order Compactification," Pacific J. Math. 118 (1985) 159-163.

[7] D. Kent and T. Richmond, "Ordered Compactification of Totally Ordered Spaces," Internat. J. Math. $\&$ Math. Sci. 11 (1988) 683-694.

[8] , "Separation Properties of the Wallman Ordered Compactification," Internat. J. Math. O Math. Sci. 13 (1990) 209-222.

[9] T. McCallion, "Compactifications of Ordered Topological Spaces," Proc. Camb. Phil. Soc. 71 (1972) 463-473.

[10] L. Nachbin, Topology and Order, Van Nostrand, New York Math. Studies, 4, Princeton, N.J. (1965). 


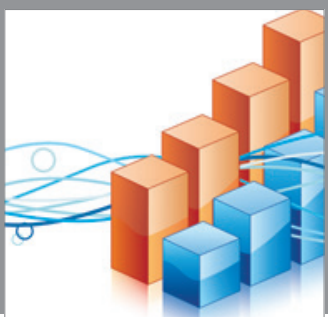

Advances in

Operations Research

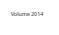

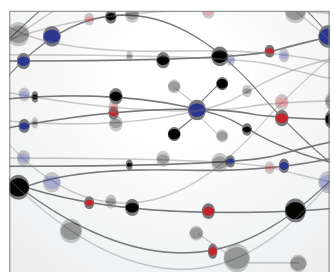

\section{The Scientific} World Journal
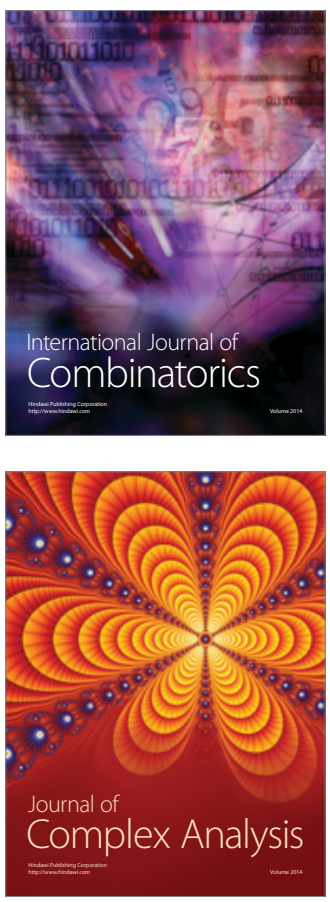

International Journal of

Mathematics and

Mathematical

Sciences
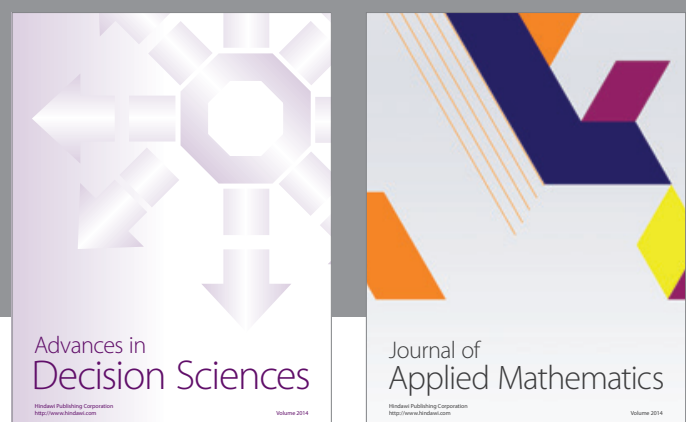

Journal of

Applied Mathematics
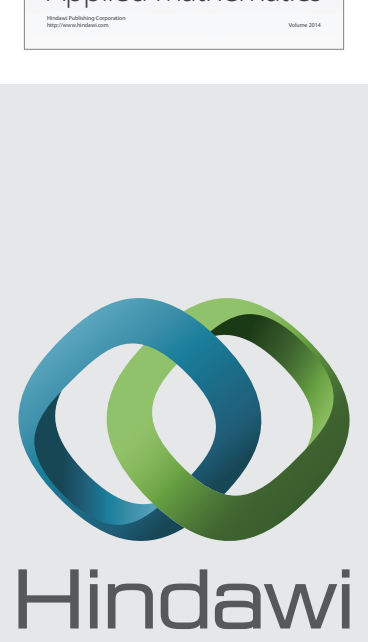

Submit your manuscripts at http://www.hindawi.com
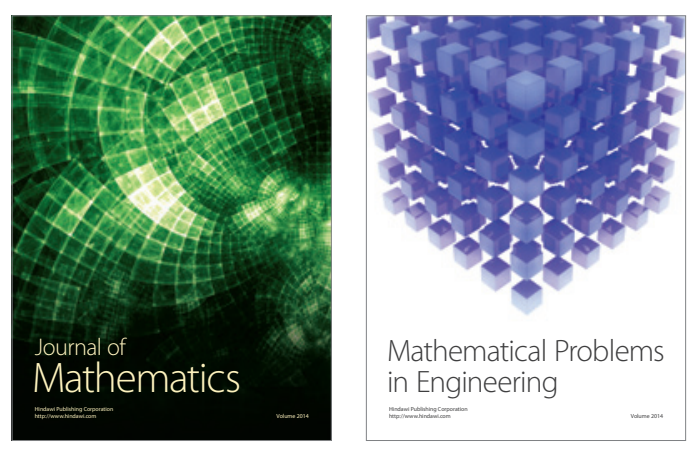

Mathematical Problems in Engineering
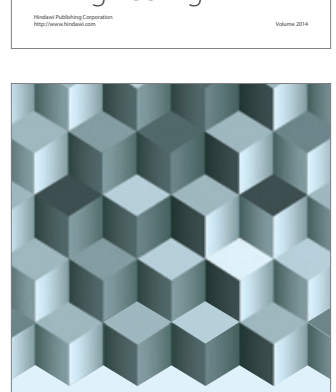

Journal of

Function Spaces
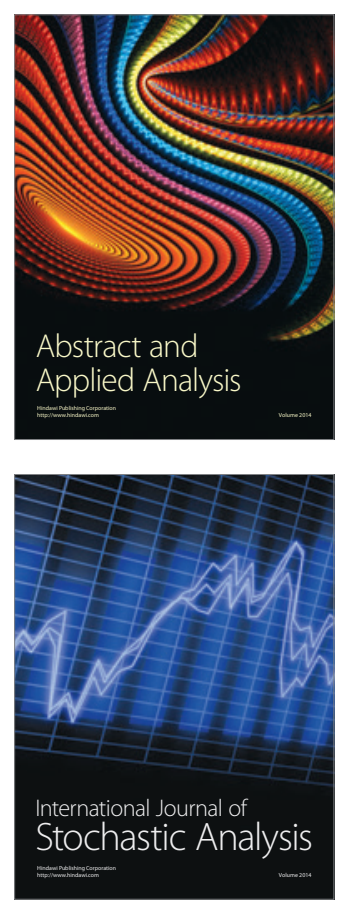

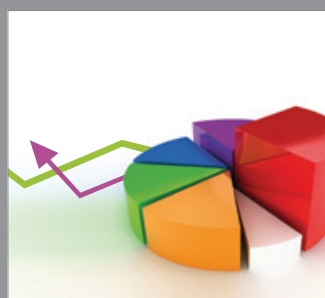

ournal of

Probability and Statistics

Promensencen
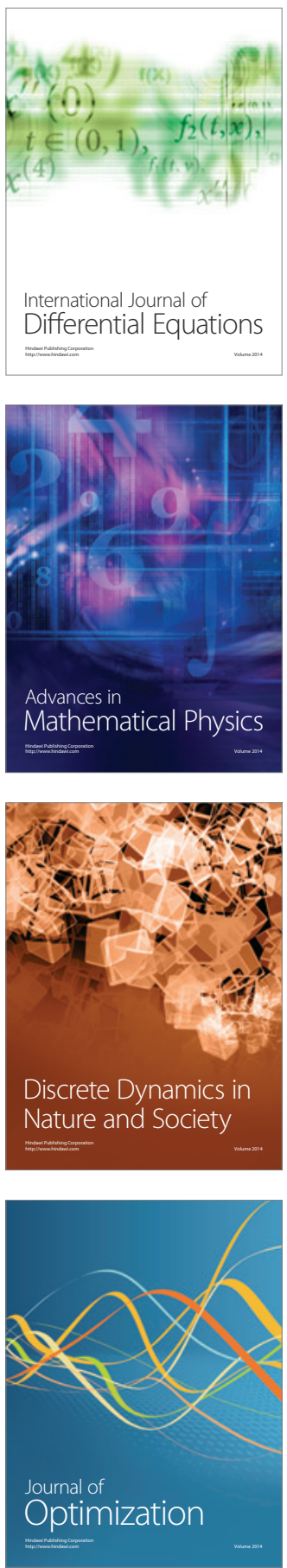